\title{
Bioinformatics and horticulture
}

\section{Opinion}

Bioinformatics is an interdisciplinary field that utilizes mathematics, engineering, biology, statistics and computer science to analyze biological data and solve complex biological problems. With the development of Human Genome Project, the data of biology increased tremendously. There was a growing need to develop a system that could measure, analyze and track this data effectively. This led to the birth of Bioinformatics which provided tools that helped in developing algorithms and software to record and analyze biological data. This includes information and characteristics of genes, proteins, drug ingredients and metabolic pathways. The key research areas in Bioinformatics are:
i. Sequence Analysis,
ii. Genome Annotation,
iii. Computational Evolutionary Biology,
iv. Analysis of Mutations in Cancer,
v. Virtual Evolution,
vi. High-throughput Image Analysis.

The onset of research in the field of Sequence Analysis and Genome Annotation has played a significant role in the area of crop improvement. An extremely large amount of genomics data is available from plants and animals due to the tremendous improvements in the field of Bioinformatics. The function of different genes in the plant and the factors affecting these genes can be predicted. This information has helped scientists to produce enhanced species of plants which are resistant to diseases, drought, herbicides, and pesticides. Plant genomics can thus help in efficient exploitation of plants as biological resources. The identification of loci of advantageous genes is a key step in breeding crops that are optimized for greater yield, costefficiency, quality, and disease resistance. By mapping the genome for Bacillus thuringiensis (a bacteria that increases soil fertility and protects the plants from pests), scientists were able to incorporate these genes into the plant which made them insect resistant. Corn, cotton, and potatoes have been made insect resistant using this methodology. Bioinformatics has also enabled the scientists to improve nutritional quality of the plants. Researchers have been
Volume 2 Issue I - 2018

\author{
Shobha Shouche, Sachin Rahangadale \\ Department of Bioinformatics, Govt Madhav Science PG \\ College, India
}

Correspondence: Shobha Shouche, Department of Bioinformatics, Govt Madhav Science PG College, Ujjain, Madhya Pradesh, India, Email shobha.shouche@gmail.com

Received: July 23, 2017| Published: January 22, 2018

successful in inserting genes in the genome of rice to increase Vitamin A levels. The genetically modified rice contains more Vitamin A, a Vitamin which is essential to maintain healthy eyes, has helped in reducing the blindness rate worldwide. Some varieties of cereal have been modified to be drought/submergence resistant and enhanced to grow in infertile soils. Bioinformatics tools are also indispensable to agriculture and horticulture from the climate change perspective.

The current climate models that effect biological diversity require improvements to become more realistic and sound. The increasing levels of Carbon dioxide emissions are alarming and to solve this problem, genomes of microbes or plants that use Carbon dioxide as their sole carbon source are being studies. Such kinds of studies require biologists, ecologists and bioinformatics experts. Plants are the basis of life on Earth and are made up of complex biological processes. The tools of Bioinformatics are vital to carry out a systematic functional analysis and make ground-breaking discoveries. Different computational tools are being used and developed for comparative genomics. The most popular tools that are used by researchers are UCSC browser, Ensemble, Map View, VISTA, and Blue Jay Genome Browser. Bioinformatics has opened up vast areas in the field of enhancing the quality and quantity of agricultural production, which researchers are only beginning to discover.

\section{Acknowledgements}

None.

\section{Conflict of interest}

The author declares no conflict of interest. 